\title{
Análisis de la Percepción del Docente en Tres Universidades Chilenas tras la Implementación del Currículum Basado en Competencias
}

\author{
Miguel A. Bustamante ${ }^{(1,2) \star}$, María del C. Lapo ${ }^{(2)}$, Claudio H. Oyarzún ${ }^{(3)}$, Roberto M. Campos ${ }^{(4)}$ \\ (1) Univ. de Talca, Campus Lircay. Avenida Lircay S/N, Talca, Chile. (e-mail: mabu@utalca.cl) \\ (2) Facultad de Ciencias Económicas y Administrativas de la Universidad Católica de Santiago de \\ Guayaquil, Av. Carlos Julio Arosemena Km. 11/2 vía Daule, Guayaquil, Ecuador. \\ (e-mail: maria.lapo@cu.ucsg.edu.ec) \\ (3) Inst. de Investigación y Desarrollo Educacional, IIDE, Univ. de Talca. Campus Lircay. Avenida Lircay \\ S/N, Talca - Chile. (e-mail: cloyarzun@utalca.cl) \\ (4) EGEU Business School, Eliodoro Yañez 869, Providencia Santiago, Chile. (e-mail: rcampos@utalca.cl) \\ * Autor a quien debe ser dirigida la correspondencia
}

Recibido Ene. 30, 2017; Aceptado Mar. 20, 2017; Versión final May. 4, 2017, Publicado Ago. 2017

\begin{abstract}
Resumen
El presente trabajo determina las percepciones docentes que caracterizan a los profesores de tres Universidades chilenas frente a la implementación de la educación con enfoque en competencias. Se analizaron los relatos libres y espontáneos de inteligibilidad de la práctica docente de una muestra de 97 académicos de planta de dichas universidades. El análisis se realizó mediante métodos cualitativos de recogida de datos y la técnica de análisis de contenido para identificar categorías de palabras, principios orientadores y criterios para caracterizar las percepciones de los profesores. Aunque se observan coincidencias de percepciones en las categorías guiar y aportar, se presentan diferencias en cómo los profesores se relacionan con los principios orientadores. Este último factor depende de la profundidad y tiempo que las respectivas Universidades llevan implementando la enseñanza con enfoque en competencias. Los profesores se enfocan en la participación y personalización de la enseñanza, la motivación, desarrollo de la autoestima e interacción. Además, aunque internalizan el contexto, en una de las universidades no se vincula la globalización a sus actividades, centrándose en los lineamientos institucionales y en los de la enseñanza tradicional por objetivos.
\end{abstract}

Palabras clave: enseñanza y formación; formación profesional; práctica pedagógica; método de enseñanza; competencias

\section{Analysis of the Teaching Perception after the Implementation of the Skills-Based Curriculum in Three Chilean Universities}

\begin{abstract}
This study shows the teaching perceptions that characterize professors of three Chilean universities that faced the implementation of a competency-based teaching approach. Free and spontaneous accounts on the teaching practice intelligibility were analyzed in a sample of 97 tenured professors from these universities. The analysis was carried out using qualitative methods of data collection and the content analysis technique to identify categories of words, guiding principles and criteria for identifying the perceptions of the professors. Although there are coincidences of perceptions in the guide and contribute categories, there are differences on how professors relate to the guiding principles. The latter concept depends on the depth and time that the each university has been implementing the competency-based approach. Professors focus on the participation and personalization of teaching, the motivation, the development of self-esteem and interaction. Also, although they internalize the context, in one of the universities globalization is not linked to its activities, focusing on the institutional guidelines and on those of the traditional teaching by objectives.
\end{abstract}

Keywords: teaching and training; professional training; teaching practice; teaching methods; skills 


\section{INTRODUCCIÓN}

El concepto de competencias definido como holístico, polisémico y sistémico (Fernandez-Elgueta y EstradaGoic, 2016) identifica genéricas, transversales, específicas (Germain, et.al., 2016; Giménez, 2016) instrumentales y sistémicas (Muñoz-Osuna, et al., 2016), que giran en torno a procesos complejos de desempeño idóneo (Robles-Haros y Esteves-Nenninger, 2016) y que se materializan en un conjunto de asignaturas (Sutcliffe, et al., 2005) para ser desarrolladas en forma evolutiva dentro de un proceso de enseñanza aprendizaje activo y autónomo (Icarte y Labate, 2016), principalmente en manos del estudiante (Lachiver, 2010).

Es el estudiante quien asumen una lógica profesional y da cuenta de la integralidad, adaptabilidad, transferencia y activación de recursos diversificados y complejos aplicables a contextos laborales, disciplinares y socio-laborales diversos (García, 2011; De Miguel, 2005 b). Desde esta perspectiva, "el profesor es guía, facilitador... para ayudar al alumno a construir su propio conocimiento" (Legault, 2012; Camargo y Pardo, 2008) haciendo indispensable en los profesores, una renovación de sus conocimientos (Castro, 2015; Carrera et al., 2012), en consecuencia, debe ser capacitado en este nuevo y complejo rol (Cisterna, et al., 2016), porque el profesorado y sus competencias son claves en la innovación y cambio que se propone (Mas-Torello y Olmos-Rueda, 2016) y porque enseñar es un acto socioeducativo complejo (Diaz et al. 2010) que debe conciliar contextos, contenidos y esfuerzo pedagógico para logar un aprendizaje activo (García et al., 2016; Lopez, et al., 2016; López y Nelson, 2003; Eraut, 1994). Por otra parte, desde la lógica de la certificación y a partir de parámetros internacionales (Knust y Gómez, 2009), el docente es un gestor y no un transmisor de conocimientos (Pavié, 2011). A él le corresponde definir lineamientos y objetivos de trabajo reflexivos, responsables, críticos, innovadores y versátiles (Pey y Chauriye, 2011), para estimular la iniciativa, producir aprendizaje y desarrollar capacidades y talentos que deben ser validados en el colectivo social (Zabalza, 2012).

El docente es entonces un experto (Perrenoud, 2001), que tiene los conocimientos, motiva y gestiona los recursos pedagógicos para guiar el aprendizaje de sus estudiantes (Coronado y Estupiñán, 2012), es un conductor del ejercicio profesional de sus aprehendientes (Ruay, 2010), e involucra su participación activa y comprometida como docente (Bustamante, et al., 2016). En consecuencia, el rol del docente comporta subjetividad (Bustamante, et al., 2015) para comprender y dar sentido a una identidad y a una profesionalidad del quehacer académico a través del análisis de la práctica singular de quien enseña (Lopez, et al., 2016; Checchia, 2009; López y Nelson, 2003; Eraut, 1994).

El método de análisis de actividades (Barbier, 1999), delimita el perfil competencial necesario para que el profesorado desarrolle adecuadamente sus nuevas funciones. (Mas-Torello y Olmos-Rueda, 2016), porque el análisis de actividades es una herramienta de co-construcción de realidades que da pie a la inteligibilidad y la toma de conciencia de competencias complejas así como al desarrollo de nuevos modelos mentales (Johnson-Laird, 1983) que hacen sentido a cada docente en su propia "singularidad" y donde "auto formación" y "experiencia" se complementan en un marco de reflexión, análisis y toma de conciencia de los factores clave que conforman sus prácticas docentes en singularidad (Bustamante et al., 2016; Bustamante et al., 2015; De Miguel, 2006) y que le permitirán transferir unos aprendizajes, antes descontextualizados, a situaciones ahora cercanas a la realidad (Giménez, 2016).

El análisis de práctica es un método de reflexión evolutivo (Giménez, 2016) sobre el ejercicio de actividades, en este caso docentes, que contribuye a la toma de conciencia de lo realizado y al mejoramiento de la capacidad de enseñar sobre la base de una combinación dinámica de atributos (Casado -Molina y Cuadrado-Mendez, 2014). El método permite, mediante el razonamiento crítico, la inteligibilidad de lo realizado, propicia la generación de los cambios de mentalidad que sean pertinentes a cada quien, mediante el uso de la reflexión sobre la propia práctica (Crisol, 2010), todo ello, para facilitar el aprendizaje, el auto conocimiento y por este medio, el de los alumnos en un espacio de autonomía y libertad (García et al., 2008), que crea el docente y que da sentido de realidad al entramado de significaciones del proceso de enseñanza - aprendizaje (Patiño-Garzón y Rojas-Betancur, 2009; De Miguel, 2006). En el análisis de la práctica, se reconoce una cierta lógica de sentido "histórico - cultural" del docente (Lopez, et al., 2016; Barbier, 1999; Eraut, 1994) que habla de "habitus" y del paradigma "psico-analítico" de un proceso de transferencia y contra-transferencia de un aprendizaje continuo, que conlleva lo "experimental" y que suponen una lógica positivista con neutralidad del animador en un ciclo que busca la objetividad de relaciones, pero que sin embargo, ocurre en un contexto de causalidad multidimensional con la realidad (Cardoso, et al., 2013).

La reflexión de la acción resulta entonces ser vital para el docente, en tanto le permite concebir en profundidad el proceso de enseñanza (Diaz et. al., 2010; López y Nelson, 2003), lo que trasunta en saber, sea que esté basada en las experiencias o en el conocimiento prescrito (teórico o doctrinario), pero que sin embargo, ocurre 
en continuidad (Muñoz, 2012) avanzando desde la acción al resultado, pero mediado por la reflexión como elemento intelectual que nace de la experiencia (Pedraja, 2012). Este proceso de análisis de la práctica, permite que la reflexión termine siendo educativa y disponga al aprendiente, en este caso del propio profesor, al cambio progresivo del sentido que dará a sus experiencias futuras en el acto de enseñar (Bustamante, 2008; De Miguel, 2006) con base más allá de los conocimientos previos o habilidades prácticas sino que orientándose a satisfacer requerimientos complejos de un contexto particular como de la sociedad en general (Viquez-Moreno, et al., 2015).

Se supone, en consecuencia, que para que una persona cambie su práctica, es preciso que el nuevo sistema de creencias no unívoco, multidimensional y complejo de valores (Morales y Varela, 2015), domine su percepción individual de la realidad en la cual la persona se desempeña y que el saber teórico previo mute en una nueva acción (Alonso-Martin, 2010; Camargo y Pardo, 2008; López y Nelson, 2003; Eraut, 1994) de modo que este cambio modifique las prácticas habituales e incorpore aptitudes, idoneidad y pericia (Clavijo, 2015), todas en conciencia y dominio, generando nuevos modelos mentales (Johnson-Laird, 1977) para que la práctica nueva e innovadora sea validada por los pares y finalmente sea asumida como la nueva regla que regulará y validará el nuevo saber (De Miguel, 2005a; De Miguel, 2005b; Popper, 2005, Popper, 1994).

Es así como, el Sistema de Educación Superior en Chile, a través del Consejo de Rectores de las Universidades Chilenas (CRUCH, 2015), adscribió a los ejes del proceso de Bologna (1999) y formuló los lineamientos del Sistema de Créditos Transferibles, SCT- Chile, sistema que sentó las bases para la homologación de los procesos formativos y el reconocimiento del esfuerzo de aprendizaje en términos de movilización de saberes (García, 2011; Puig y García, 2007), todo lo cual supone autonomía y desarrollo de habilidades esenciales (Lopez, et al., 2016; Denyer et al., 2007; Eraut, 1994) así como también, la necesaria adecuación a la realidad de quien enseña saberes complejos (Pey y Chauriye, 2011) y que, cuando son activados, conforman las denominadas competencias profesionales (Lachiver, 2010)

A nivel sistémico, las Universidades han incorporado progresivamente innovaciones, conductas de trabajo y en modos de acción (Turcio-Ortega y Palacios-Alquisira, 2015) en el actuar de sus organizaciones así como en sus planes de formación profesional incluyendo transformaciones de sus sistemas de enseñanza e integrado los ejes de participación, unicidad, adaptabilidad y coherencia interna y externa del aprendizaje (Ettayebi et al., 2008). De acuerdo con estos ejes, el estudiante es un agente activo en el desarrollo de su propio aprendizaje y es capaz de movilizar y combinar sus recursos, personales y contextuales, para lograr el ejercicio de una actividad en excelencia (Lopez, et al., 2016; Lachiver, 2010; Denyer et al., 2007; De Miguel, 2006; Eraut, 1994).

\section{METODOLOGÍA}

A partir de la conceptualización analizada y, sobre la base de la incorporación progresiva en las Universidades del proceso de enseñanza con enfoque en competencias, surgen a lo menos dos interrogantes esenciales que este trabajo pretende analizar: ¿Cuáles son las percepciones del quehacer pedagógico que se genera en los docentes a partir del cambio del modelo de enseñanza por objetivos al basado en competencias? y, ¿En qué términos se pueden caracterizar las Universidades que han asumido la implementación del modelo de enseñanza con enfoque en competencias?

El presente trabajo busca, en consecuencia, identificar las percepciones docentes que caracterizan la práctica de los profesores en tres universidades que implementan la enseñanza con enfoque en competencias. Para ello, se aplicó una metodología cualitativa-cuantitativa, recogiendo información tal como se da en su contexto natural, sin realizar ningún tipo de manipulación sobre ninguna de las variables objeto de estudio (Hernandez, et al., 2006). La fuente de datos primarios se obtuvo de entrevistas de explicitación escritas (relatos espontáneos) y del análisis de las significaciones implícitas en dichos relatos mediante el análisis de contenidos (Abela, 2013; Fernández, 2002).

Para el estudio de campo (García-Cabrero, et. al., 2008) se recurrió a tres universidades chilenas. La Universidad (A) que oficializó su Proyecto Educativo Institucional (PEI) el año 2010 adoptando la metodología de enseñanza con enfoque en competencias. La Universidad (C) que se sumó a estos lineamientos educativos a contar del año 2004 mediante innovaciones en los currículum adoptándolo el año 2008, finalmente, la Universidad (B), que es la más antigua en este tipo de innovaciones, que completa ya diez años de implementación, con innovaciones y adecuaciones progresivas al modelo de enseñanza con enfoque en competencias.

Se realizó un muestreo no probabilístico por conveniencia (Hernandez, et al., 2006) orientándose a la selección de aquellas unidades muestrales (docentes) que garantizaran cantidad y calidad de información relevante. El criterio de inclusión fue que los participantes fuesen docentes de cátedra y pertenecieran a la 
categoría de planta de las Universidades en estudio, totalizando 97 profesores; 34 que pertenecen a la Universidad (A), 32 a la Universidad (B) y 31 a la Universidad (C). Para extraer la información relevante desde los relatos, se utilizó la técnica del análisis de contenido (Fernández, 2002; López, 2002) identificando elementos tales como palabras y frases significativas, para ser clasificadas en variables y categorías explicativas del fenómeno en investigación.

\section{Procedimiento a seguir}

Como se indicó, el análisis interpretativo, los términos, acepciones y palabras, derivaron de los relatos personales (Ander-Egg, 2004; López y Nelson, 2003; Murcia, 2003; Gadamer, 1998; Diéguez, 1993; Bunge, 1982) que los docentes expresaron, como unidades hermenéuticas (Ángel, 2011; Arango, 2011; Barragán, 2008; Ortiz y Lanceros, 2005) frente a la innovación de la enseñanza que en sus respectivas entidades se implementó. Se identificaron términos clave a partir del lenguaje propio y espontaneo de los entrevistados, se enfocaron coincidencias - divergencias y agrupamientos conceptuales de interés para la investigación (Abela, 2013; Baeza, 2002). Se aplicó el análisis de contenido de los relatos escritos (Vattimo, 1991) y se utilizó mecanismos de definición de categorías compuestas por variables hipotéticas en vinculación con reflexiones, perspectivas y niveles de expresión para desglosar unidades de análisis significativas.

Puesto que la hermenéutica es el centro de los métodos de investigación social (Arango, 2011; Barragán, 2008; Ortiz y Lanceros, 2005), la codificación consistió en la transformación de los registros en unidades interpretables. Es decir, las unidades hermenéuticas de análisis son las que permiten interpretaciones y éstas, son definidas como aspectos metodológicos de una investigación, destinados a producir frutos hermenéuticos como una aproximación, interpretable de los fenómenos humanos que deben ser analizados en sus propios términos y traducidos al contexto en estudio (Ángel, 2011). Es por ello que en este trabajo, cuyos relatos provienen de la experiencia y percepción de los profesores entrevistados, se definen categorías y subcategorías, dando forma a unidades de registro factibles de descripción, interpretación y análisis (Santander, 2011; Ortiz y Lanceros, 2005).

La cuantificación se hizo mediante diversas modalidades de frecuencia absoluta, relativa y datos estandarizados en categorías y principios orientadores, para detectar posibles usos comunes del mismo término. (Fernández, 2002). Para la codificación en sistemas y categorías semánticas, se tuvo a la vista el marco conceptual y el significado ontológico de las palabras (Echeverría, 2007; Ortiz y Lanceros, 2005). Se aplicó Software de QSR Atlas Ti para Windows, para unificar palabras y frases, y además obtener frecuencias absolutas y relativas (en porcentajes de cobertura) para asignar los pesos o importancia dentro del total de relatos. Además se usó Microsoft Office Excel 2010 para la elaboración de gráficos, tablas, y cálculos de las codificaciones por categoría, como se esquematiza en la Tabla 1.

Tabla 1: Elementos de análisis y descriptores

\begin{tabular}{ll}
\hline Elemento & Descriptor \\
\hline Muestra & 97 relatos \\
Finalidad central de la investigación & $\begin{array}{l}\text { Determinar las concepciones docentes frente al proceso de } \\
\text { innovación curricular }\end{array}$ \\
Regla de numeración & Frecuencia estandarizada \\
Categorización & $\begin{array}{l}\text { Clasificación por significado, a través de un sistema de categorías } \\
\text { semánticas }\end{array}$ \\
Unidad de Registro & Palabra y frase \\
Unidad de Contexto & Para la palabra corresponde la frase \\
& Para la frase corresponde el tema \\
\hline
\end{tabular}

Como criterio de selectividad, se consideró que las categorías de palabras y frases alcanzaran los mayores porcentajes de cobertura y que fuesen mencionadas un mínimo de dos oportunidades para ser clasificadas en conceptos representativos de una descripción significativa (Checchia, 2009; Balcazar, 2005).

\section{RESULTADOS}

Los resultados se presentan clasificados en tres grandes apartados, primero una codificación general por categorías de palabras, una codificación por categoría de frases y una síntesis de percepciones, de acuerdo al orden en que se llevó a cabo el proceso de análisis de los datos. 


\section{Codificación general por categorías de palabras}

La Tabla 2, muestra las frecuencias absolutas y estandarizadas de las palabras más frecuentemente expresadas por los docentes de las respectivas Universidades. Se determinaron en total 21 palabras o expresiones fuerza que sintetizan las verbalizaciones espontáneas de los profesores en torno a su actividad como docentes, confirmando que efectivamente el profesor es un guía o un facilitador que ayuda al estudiante a "construir su propio conocimiento" (Lopez, et al., 2016; Legault, 2012; Lachiver, 2010; Camargo y Pardo, 2008; De Miguel, 2006).

Tabla 2: Codificación general por categoría de palabras. Frecuencias absolutas y estandarizadas

\begin{tabular}{|c|c|c|c|c|c|c|c|}
\hline & Universidad & & $A$ & & B & & $\mathrm{C}$ \\
\hline & Palabras & Frec. & Estándar & Frec. & Estándar & Frec. & Estándar \\
\hline 1 & Actividades & 95 & 2,946 & 179 & 2,522 & 30 & 0,372 \\
\hline 2 & Aprendizaje & 66 & 1,743 & 145 & 1,783 & 37 & 0,745 \\
\hline 3 & desarrollo (ar) & 23 & $-0,041$ & 94 & 0,674 & 32 & 0,479 \\
\hline 4 & Contenidos & 50 & 1,079 & 64 & 0,022 & 4 & $-1,011$ \\
\hline 5 & Experiencias & 49 & 1,037 & 65 & 0,043 & 13 & $-0,532$ \\
\hline 6 & formación/formar & 33 & 0,373 & 114 & 1,109 & 52 & 1,543 \\
\hline 7 & Conocimiento & 27 & 0,124 & 77 & 0,304 & 6 & $-0,904$ \\
\hline 8 & Manera/proceso & 26 & 0,083 & 42 & $-0,457$ & 33 & 0,532 \\
\hline 9 & Contexto & 26 & 0,083 & 41 & $-0,478$ & 2 & $-1,117$ \\
\hline 10 & Competencias & 12 & $-0,498$ & 94 & 0,674 & 38 & 0,798 \\
\hline 11 & Trabajar & 12 & $-0,498$ & 106 & 0,935 & 54 & 1,649 \\
\hline 12 & evaluación (es) & 13 & $-0,456$ & 26 & $-0,804$ & 3 & $-1,064$ \\
\hline 13 & Responsabilidad & 14 & $-0,415$ & 35 & $-0,609$ & 17 & $-0,319$ \\
\hline 14 & Enseñar & 12 & $-0,498$ & 44 & $-0,413$ & 18 & $-0,266$ \\
\hline 15 & buscar/profesional & 4 & $-0,830$ & 30 & $-0,717$ & 51 & 1,489 \\
\hline 16 & metodología/método & 8 & $-0,664$ & 24 & $-0,848$ & 7 & $-0,851$ \\
\hline 17 & Docencia & 6 & $-0,747$ & 67 & 0,087 & 51 & 1,489 \\
\hline 18 & Gestión & 4 & $-0,830$ & 24 & $-0,848$ & 11 & $-0,638$ \\
\hline 19 & Apoyar & 4 & $-0,830$ & 19 & $-0,957$ & 8 & $-0,798$ \\
\hline 20 & Implementación & 4 & $-0,830$ & 16 & $-1,022$ & 6 & $-0,904$ \\
\hline \multirow[t]{3}{*}{21} & Crear & - & - & 8 & $-1,196$ & 1 & $-1,170$ \\
\hline & Promedio & 24 & & 63 & & 23 & \\
\hline & Desviación estándar & 24,1 & & 46 & & 18,8 & \\
\hline
\end{tabular}

Si se analizan los valores estandarizados más altos, a la derecha de la curva normal, de la Tabla 2 y de la Figura 1, se observa que en general, los docentes mencionan las palabras: actividades que alcanza entre 2,946 y 2,522 puntos estándar, aprendizaje que muestra 1,743 y 1,783 puntos estandarizados, seguido de los términos formación/formar con 1,109 y 1,543 puntos estándar.

En el caso de la Universidad A, las palabras que destacan son actividades, aprendizaje, contenidos $y$ experiencias. En tanto que, en la Universidad B, los profesores destacaron las expresiones, actividades, aprendizaje y formación/formar. Por último, los académicos de la Universidad C manifestaron como términos orientadores de su accionar las palabras trabajar, formación/formar, buscar/profesional y docencia.

Sobre la base de los datos estandarizados, se confirma que los docentes de pregrado de la Universidad A, presentan un perfil de enseñanza enfocado en la enseñanza apoyados por las palabras conocimiento, contexto, manera/proceso, sin embargo, entre las palabras menos utilizadas se encuentran, implementación, apoyar, gestión, docencia y metodología/proceso, términos que trasuntan y ponen de manifiesto que el enfoque por competencias está aún en ciernes en esta institución. Es decir las actividades de enseñanza de los profesores, se comienzan a acercar progresivamente al enfoque por competencias transfiriendo a los futuros egresados los entrenamientos que éstos necesitarán a futuro para desenvolverse, en su actividad profesional (De Miguel, 2006; De Miguel, 2005 a; De Miguel, 2005 b). 
Los docentes de pregrado de la Universidad B, muestran conductas que, en términos de los valores estandarizados, expresan la valoración que dan a su accionar docente, enfocándose en el desarrollo de aprendizajes, en cumplir con los objetivos y contenidos y, al mismo tiempo, enriquecer el proceso de enseñanza mediante la experimentación y el logro a través del trabajo con enfoque en competencias, que se expresa en los términos desarrollo, docencia, trabajar y competencias (Checchia, 2009). Entre los términos menos utilizados se mencionan crear, implementación, apoyar y gestión. Sin embargo, se observa que el foco de percepciones de los docentes está dado por la relevancia que le asignan a la categoría formación/formar, desde los contenidos y la experiencia que, desde la educación por competencias tiene su foco centrado en los estudiantes, sin perjuicio de sostener y tener a la vista, la lógica tradicional de la enseñanza por contenidos (Coronado, 2012).

Los académicos de la Universidad C, muestran grados de dispersión mayor de los puntajes estandarizados. Eventualmente esto puede ser el resultado, por una parte, porque las frecuencias indicadas son menores y, por otra, porque los docentes de esta institución se identifican, desde hace menos tiempo, con el enfoque de convergencia europea de la enseñanza superior (De Miguel, 2006; De Miguel, 2005 a). Los principales significados provienen de las palabras, trabajar, formación/formar, buscar/profesional y docencia, seguidas de los términos aprendizaje, manera/proceso, desarrollo y competencias. Entre las palabras menos mencionadas se cuentan, crear, contexto y contenidos en cuya secuencia se ubican también con escasa frecuencia y bajo valor estándar los términos implementación, apoyar y gestión. Se observa la búsqueda de nuevas funciones dentro del rol académico y la mejora de su docencia, internalizando nuevas prácticas para ser habilitados según este nuevo paradigma (Díaz, et al., 2010).

Integrando gráficamente la información descrita en la Tabla 2, la Fig. 1 pone en evidencia los términos más recurrentes mencionados por los docentes de cada una de las tres Universidades, haciendo posible una primera caracterización agregada de las percepciones. Es interesante observar cómo dos instituciones tan disímiles como la Universidad $\mathrm{B}$, de raigambre pública y la $\mathrm{C}$, de origen privado, coinciden en el grado de utilización del término competencias, considerando que la primera, lleva años implementando dicha metodología de enseñanza respecto de la segunda que recientemente se ha incorporado a dicho enfoque de enseñanza (García-Cabrero, et al., 2008).

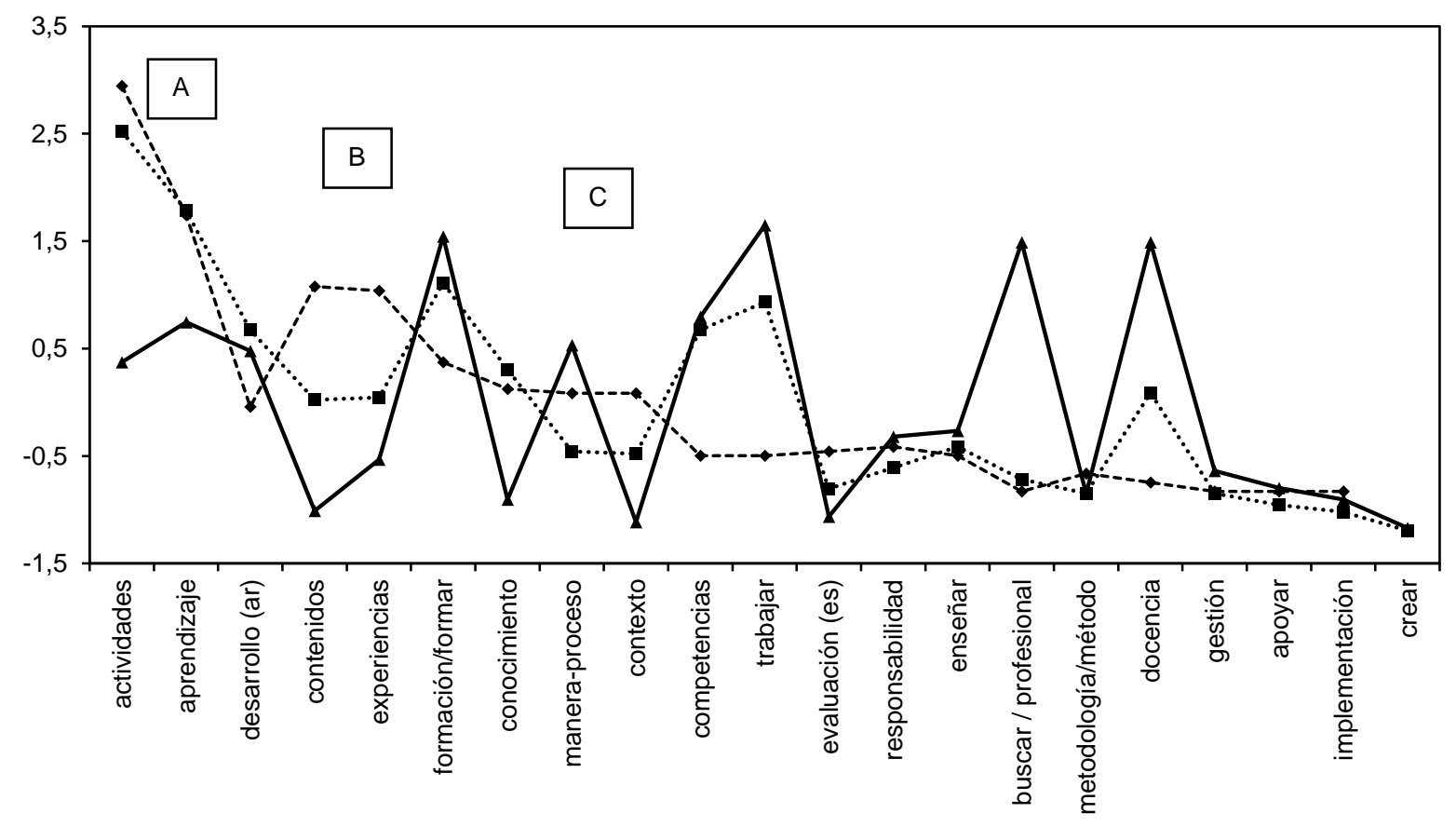

Fig. 1: Concepciones docentes más frecuentes. Valores estandarizados

Así mismo, se observan coincidencias entre las Universidades A y B, en este caso ambas de origen público, cuando sus docentes mencionan las palabras actividades, aprendizaje, en clara referencia al enfoque de enseñanza por competencias en combinación con los métodos tradicionales de enseñanza por contenidos. Las restantes palabras, tales como evaluación (evaluadores), responsabilidad, enseñar y metodología/ método, entre otras, conforman el arsenal permanente de concepciones docentes que animan la conducta de los profesores y señalizan su comportamiento que, dentro del sistema universitario, ha validado el quehacer de los académicos (Lopez, et al., 2016; Lachiver, 2010; Knust y Gómez, 2009; Eraut, 1994). 


\section{Codificación por categoría de frases}

La Tabla 3, consolida los principios orientadores y las frecuencias absolutas y estandarizadas de las percepciones docentes.

Tabla 3: Categorías y principio / orientador por Universidad. Frecuencias absolutas y estandarizadas

\begin{tabular}{|c|c|c|c|c|c|c|c|c|c|c|}
\hline $\begin{array}{l}\text { Categorías } \\
\text { Universidad }\end{array}$ & Aportar & ender & Capacitar & Educar & Enseñar & Estructurar & Evaluar & Guiar & Planificar & Total \\
\hline \multirow{2}{*}{ Universidad A } & 12 & 15 & 23 & 33 & 14 & 28 & 33 & 50 & 27 & 235 \\
\hline & $-1,02$ & $-0,82$ & $-0,31$ & 0,34 & $-0,89$ & 0,02 & 0,34 & 1,44 & $-0,05$ & \\
\hline \multirow[t]{2}{*}{ Universidad B } & 21 & 40 & 30 & 15 & 40 & 19 & 1 & 42 & 4 & 212 \\
\hline & $-0,44$ & 0,79 & 0,15 & $-0,82$ & 0,79 & $-0,56$ & $-1,73$ & 0,92 & $-1,53$ & \\
\hline \multirow[t]{2}{*}{ Universidad C } & 8 & 44 & 24 & 54 & 56 & 49 & 36 & 17 & 14 & 302 \\
\hline & $-1,28$ & 1,05 & $-0,24$ & 1,70 & 1,83 & 1,37 & 0,53 & $-0,69$ & $-0,89$ & \\
\hline \begin{tabular}{l}
\multicolumn{2}{c}{ Total } \\
Principio /
\end{tabular} & 41 & 99 & 77 & 102 & 110 & 96 & 70 & 109 & 45 & 749 \\
\hline \multirow{3}{*}{$\begin{array}{l}\text { Principio / } \\
\text { orientador } \\
\text { Universidad A }\end{array}$} & $\begin{array}{c}\text { Actividad y } \\
\text { Participación }\end{array}$ & \multicolumn{2}{|c|}{$\begin{array}{l}\text { Motivación y } \\
\text { Autoestima }\end{array}$} & \multicolumn{2}{|c|}{$\begin{array}{l}\text { Aprendizajes } \\
\text { Significativos }\end{array}$} & balización & \multicolumn{2}{|c|}{ Personalización } & \multicolumn{2}{|l|}{ Interacción } \\
\hline & 60 & \multicolumn{2}{|c|}{17} & \multicolumn{2}{|c|}{26} & 71 & \multicolumn{2}{|c|}{60} & 1 & 235 \\
\hline & 0,91 & \multicolumn{2}{|r|}{$-1,21$} & \multicolumn{2}{|c|}{$-0,77$} & 1,45 & \multicolumn{2}{|c|}{0,91} & -2 & \\
\hline \multirow[t]{2}{*}{ Universidad B } & 22 & \multicolumn{2}{|r|}{44} & \multicolumn{2}{|c|}{53} & 36 & \multicolumn{2}{|c|}{24} & 33 & 212 \\
\hline & $-0,97$ & \multicolumn{2}{|r|}{0,12} & \multicolumn{2}{|c|}{0,56} & $-0,28$ & \multicolumn{2}{|c|}{$-0,87$} & $-0,42$ & \\
\hline \multirow[t]{2}{*}{ Universidad C } & 64 & \multicolumn{2}{|r|}{34} & \multicolumn{2}{|c|}{29} & 65 & \multicolumn{2}{|c|}{69} & 41 & 302 \\
\hline & 1,1 & \multicolumn{2}{|r|}{$-0,38$} & \multicolumn{2}{|c|}{$-0,62$} & 1,15 & \multicolumn{2}{|c|}{1,35} & $-0,03$ & \\
\hline Total & 146 & & 95 & 10 & & 172 & & 153 & 75 & \\
\hline
\end{tabular}

Como se puede observar, las funciones del trabajo académico descrito en los relatos de los profesores, aunque se ubican a cada lado de la normal estandarizada, son comunes a todos los docentes entrevistados. Por una parte, los docentes de la Universidad A, declaran su propósito natural de guiar, educar, evaluar y estructurar, alcanzando estos términos puntuaciones positivas en valores estandarizados, sin embargo, con estándar negativo se ubican las palabras enseñar, aprender, capacitar y planificar; poniendo en juego diversas expresiones de docencia asimiladas como esenciales en la práctica pedagógica y cuyo destino natural es facilitar la tarea de aprender de sus estudiantes (Zabalza, 2012).

Desde la perspectiva de los docentes de la Universidad B, las palabras con mayores valores estandarizados fueron guiar, enseñar y aprender, caracterizando a estos docentes como claramente orientados por el enfoque de enseñanza basado en competencias. Sin embargo, dada la naturaleza de las mediciones estándar (López y Nelson, 2003), los términos menos frecuentes resultaron ser evaluar, planificar educar y estructurar, poniendo en evidencia que, aunque el enfoque hacia las competencias parece bien orientado, se confronta sistemáticamente con el enfoque de la enseñanza por objetivos de aprendizaje tradicional (Ortiz y Lanceros, 2005).

Para el caso de la Universidad C, las frecuencias estandarizadas señalizan el natural enfoque de enseñanza por objetivos que se expresa en los términos enseñar, estructurar, educar y aprender, en contraste con los términos menos frecuentes que fueron aportar, planificar, guiar y capacitar, evidentemente, éstos últimos, relacionadas con el enfoque basado en competencias. En consecuencia, en esta Universidad es evidente la existencia de una cierta dicotomía en la percepción que los profesores hacen de su labor docente, tal vez, por encontrarse en un estado de evolución inicial dada su reciente incorporación al enfoque de la enseñanza con énfasis en competencias.

Desde la perspectiva de los principios orientadores y en general, con las mayores frecuencias se mencionan las tareas globalización, personalización y actividad y participación, todas representativas de funciones clave del proceso de enseñanza y que actúan como inductoras o causales, de la motivación y autoestima para lograr aprendizajes significativos en los estudiantes, todas ellas, en clara referencia a un proceso general de enseñanza - aprendizaje (Puig y García, 2007).

Los docentes de la Universidad A definen su labor en los términos con estándar superior en las categorías: globalización, personalización y actividad y participación, en tanto que entre los menos frecuentes se ubican las palabras motivación y autoestima y aprendizajes significativos. Percepciones que evidencian una cierta dicotomía que confronta la fuerza del enfoque por competencias respecto de la enseñanza por objetivos del modelo tradicional. Los profesores de la Universidad B, por su parte, marcan altas frecuencias estandarizadas en las categorías aprendizajes significativos y, motivación y autoestima, al tiempo que 
muestran bajas frecuencias en los términos compuestos actividad y participación, personalización e Interacción, palabras que se relacionan con la enseñanza con enfoque en competencias. Estas expresiones sintetizan cómo los profesores asumen su actividad docente en relación con el modelo de enseñanza adoptado por su institución.

Finalmente, los profesores de la Universidad C, se expresan positivamente con estándares altos a través de las categorías personalización, globalización y actividad y participación como forma de concreción de una labor docente orientada a la formación, la cual sin embargo se contrapone a las palabras menos utilizadas tales como aprendizajes significativos, motivación y autoestima e interacción. Todas estas expresiones sintetizan las percepciones de profesores que, intentando incorporar el enfoque de enseñanza basada en competencias, tienden a distanciarse de las preocupaciones de la enseñanza por objetivos tradicional, redefiniendo sus propias funciones y, como consecuencias de ello, debilitando una parte esencial del actuar docente como es el de la motivación y activación de los talentos de sus estudiantes.

En general, se observa que los profesores de las Universidades A y C coinciden en su preocupación por la globalización y actividad y participación de sus estudiantes mientas los docentes de la Universidad B centran su atención en los aprendizajes significativos de sus alumnos. En suma, los principios orientadores o categorías reflejan de manera sintética la labor de los docentes y, aunque se ubican a cada lado de la normal, representan la lógica que anima, indistintamente, la actividad de los profesores de pregrado.

\section{Síntesis de concepciones docentes}

En la Tabla 4, se analiza la estructura de palabras que explican cada uno de los principios orientadores ya identificados y se detalla, para cada una de las tres universidades, la terminología más frecuente que los docentes reportan en sus relatos.

Tabla 4: Concepciones docentes en cada Universidad

\begin{tabular}{|c|c|c|c|c|c|c|c|}
\hline $\begin{array}{l}\text { Principio/ } \\
\text { Orientador }\end{array}$ & Universidad A & Frec. & Universidad B & Frec. & Universidad C & Frec. & Total \\
\hline $\begin{array}{l}\text { Actividad y } \\
\text { Participación }\end{array}$ & $\begin{array}{l}\text { Educar, Evaluar } \\
\text { Estructurar, Guiar. }\end{array}$ & 4 & Aportar - Guiar. & 2 & $\begin{array}{c}\text { Aprender, Capacitar } \\
\text { Estructurar, } \\
\text { Planificar }\end{array}$ & 4 & 10 \\
\hline $\begin{array}{l}\text { Motivación y } \\
\text { Autoestima }\end{array}$ & Educar. & 1 & $\begin{array}{c}\text { Aprender, Capacitar } \\
\text { Enseñar, } \\
\text { Estructurar, } \\
\text { Planificar. }\end{array}$ & 5 & $\begin{array}{l}\text { Aportar, Aprender } \\
\text { Educar } \\
\text { (3) }\end{array}$ & 0 & 9 \\
\hline $\begin{array}{l}\text { Aprendizajes } \\
\text { Significativos }\end{array}$ & Enseñar, Guiar & 2 & $\begin{array}{l}\text { Aportar, Aprender } \\
\text { Enseñar, Evaluar, } \\
\text { Guiar, Planificar. }\end{array}$ & 6 & $\begin{array}{l}\text { Aportar, Educar } \\
\text { Enseñar, Evaluar, } \\
\text { Guiar, Planificar. }\end{array}$ & 6 & 14 \\
\hline Globalización & $\begin{array}{c}\text { Aportar - Aprender } \\
\text { - Capacitar, } \\
\text { Estructurar - Guiar - } \\
\text { Planificar. }\end{array}$ & 6 & $\begin{array}{c}\text { Aprender, Capacitar } \\
\text { Educar, Enseñar, } \\
\text { Planificar. }\end{array}$ & 5 & 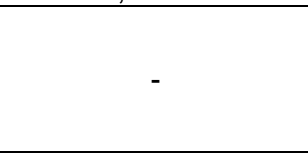 & 0 & 11 \\
\hline Personalización & $\begin{array}{l}\text { Capacitar, Evaluar, } \\
\text { Guiar, Planificar. }\end{array}$ & 4 & Capacitar, Enseñar & 2 & $\begin{array}{l}\text { Aportar, Educar } \\
\text { Estructurar, Evaluar, } \\
\text { Guiar, Planificar. }\end{array}$ & 6 & 12 \\
\hline Interacción & Evaluar & 1 & $\begin{array}{c}\text { Capacitar, Enseñar, } \\
\text { Estructurar, Guiar, } \\
\text { Planificar. }\end{array}$ & 5 & $\begin{array}{l}\text { Capacitar, Enseñar, } \\
\text { Guiar, Planificar. }\end{array}$ & 4 & 10 \\
\hline Total & & 18 & & 25 & & 23 & 66 \\
\hline
\end{tabular}

Un análisis general de los registros sintetizados muestra que los principios orientadores concentran entre 14 y 9 menciones, señalizadas en la columna total, a través de las cuales los docentes expresan la significación que les reportan sus actividades académicas. Entre éstas, la más significativa resulta ser aprendizajes significativos y la menos referida motivación y autoestima.

Del análisis efectuado a cada Universidad, los docentes manifiestan entre 18 y 25 palabras que se acumulan en la última fila (Total), como descriptivas de las acciones que realizan los profesores. Entre las menos referidas, con una frecuencia, se mencionan las palabras educar y evaluar, mientras que entre las más frecuentes mencionan las secuencias de términos aportar - aprender - capacitar, estructurar - guiar planificar y la secuencia aportar, educar - enseñar, evaluar, guiar, planificar. 
Para los profesores de la Universidad A, la categoría globalización resultó relevante y fue expresada mediante la secuencia aportar - aprender - capacitar, estructurar - guiar y planificar. Los académicos de la Universidad B por su parte, priorizaron aprendizajes significativos a través de la secuencia de palabras aportar - aprender - enseñar - evaluar - guiar y planificar. Por último los profesores de la Universidad C, mencionaron los criterios aprendizajes significativos y personalización, a través de las palabras aportar educar - enseñar - evaluar - guiar y planificar y la palabra estructurar.

\section{Caracterización docente por universidad}

De la Fig. 2, desarrollada mediante ATLAS.ti, se observan nueve de las 21 palabras mencionadas por los profesores. Con línea continua se indican las ubicadas en la parte positiva y con línea cortada las ubicadas en la parte negativa de la normal estandarizada. En general, se observan importantes similitudes entre los académicos de las Universidad A, B y C, en tanto coinciden en dos palabras clave relacionadas con el enfoque de enseñanza por competencias como son los términos aportar y guiar.

Sin embargo, un análisis más detallado permite observar que, en la Universidad A, se observan 3 palabras por debajo de la normal (capacitar, educar y evaluar), evidentemente representativas de las tareas que habitualmente realizan los docentes y que por eso mismo, pueden pasar inadvertidas. En Universidad B, 4 palabras aparecen con escasa frecuencia (planificar, estructurar, capacitar, educar), al parecer, porque sus académicos han asimilado nuevas prácticas y superado progresivamente y por sí mismos los desafíos del sistema tradicional de enseñanza por objetivo, internalizando eso sí, terminología clave para el modelo de enseñanza por competencia. Finalmente, en la Universidad C, se presenta 2 palabras entre las menos frecuentes (capacitar y aprender) lo cual puede representar cómo se han elevado las necesidades formativas de los académicos y la necesidad de ser cualificados para enseñar de acuerdo con el nuevo paradigma de la formación basada en competencias.

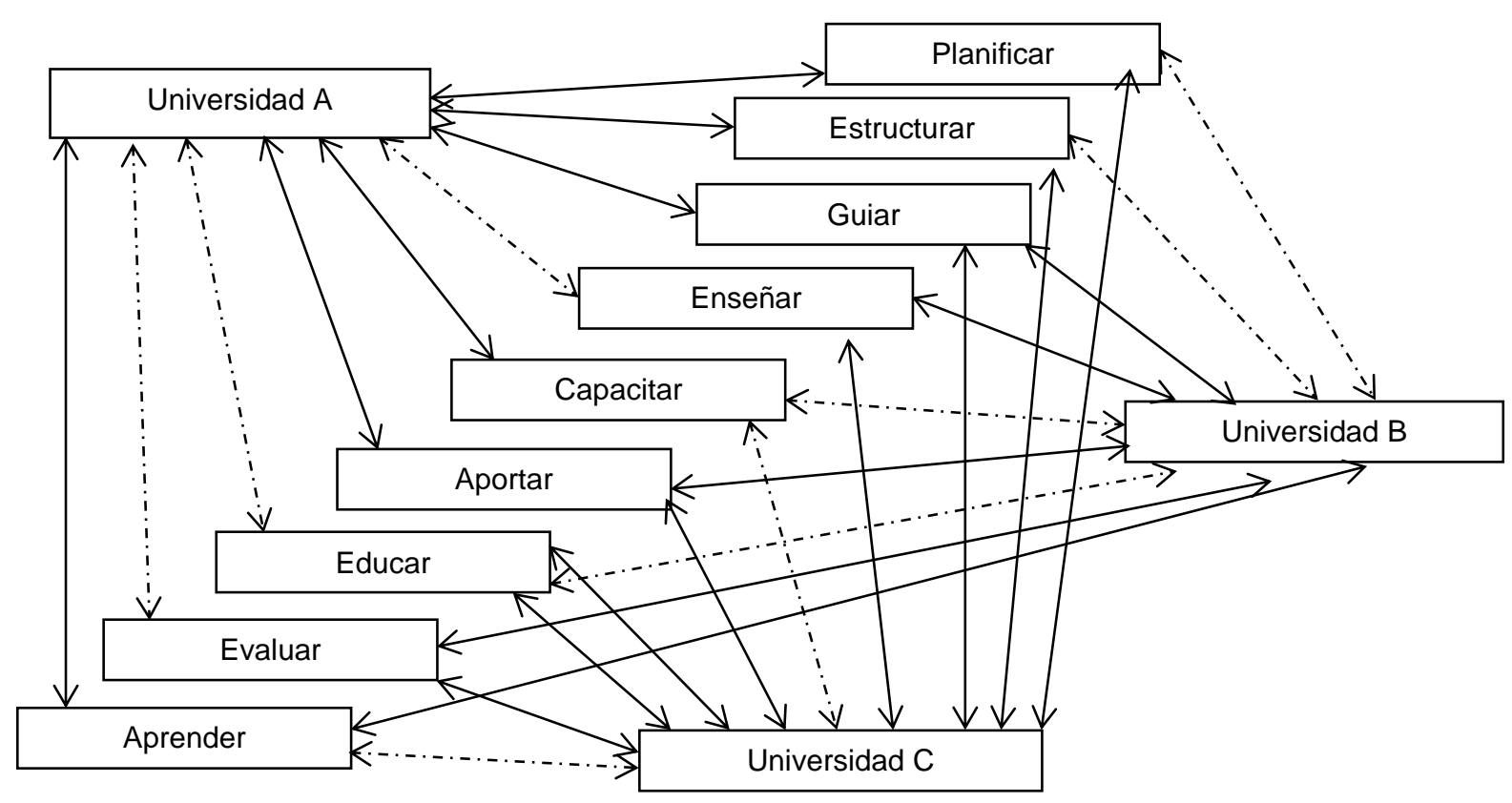

Fig. 2: Percepciones Docentes en Tres Universidades Chilenas. Salida adaptada de Software de QSR Atlas Ti

\section{Caracterización general}

En general los docentes parecen realizar un esfuerzo consciente y razonado para lograr el desarrollo de las competencias profesionales de sus alumnos a través de la personalización de los métodos de enseñanza, enmarcando su práctica en acciones de enseñanza - aprendizaje sobre la base del enfoque por competencias. Los docentes internalizan el contexto situacional en el cual se encuentran sus estudiantes de forma tal que la actividad docente desarrollada por ellos facilite el aprendizaje de los alumnos a partir de un modelo de enseñanza docente más bien constructivista. Las categorías conceptuales mencionadas y evidenciadas en la Tabla 4 y en la Figura 2, muestran cuáles son los términos o palabras a través de las cuales los profesores expresan su quehacer docente. En general, se observa que los profesores están principalmente centrados en los lineamientos nacionales de la enseñanza para la implementación de un currículum determinado. 
La enseñanza que a juicio de los profesores requieren sus estudiantes de pregrado se basa, en general, en la personalización de los métodos utilizados, por una parte, adaptados a la diversidad de los estudiantes que enfrentan así como por la existencia de algunos docentes auto motivados que facilitan la entrega de contenidos y prácticas y, a través de ello, colaboran en la absorción de dichos contenidos por parte de sus alumnos, en tanto que otros profesores, lo hacen a partir de sus capacidades de relacionamiento en trabajos en equipo, presentaciones grupales, retroalimentaciones de los pares, etc.

La actividad de planificación es una de las más recurrentes y está asociada a los principios de la formación tradicional por contenidos y que se materializa por medio de actividad y participación, aprendizaje significativo, personalización e interacción, de lo cual se puede inferir el énfasis parcial de las metodologías de enseñanza hacia el trabajo colaborativo, trabajo en equipo y motivación de grupos, lo que permite a los docentes cumplir algunas de las metas institucionales de sus respectivas Universidades. Desde una perspectiva amplia, se observa que los académicos, asocian las actividades de guiar a las de aprendizaje significativo y autoestima; entendiendo que el desarrollo de habilidades e integración de saberes de sus aprehendientes da como resultado un profesional seguro de sus conocimientos y competente en el ámbito profesional. Por otra parte, es posible afirmar que los profesores propician la personalización de su docencia, es decir, ponen en relación el aporte generacional de la juventud de sus estudiantes con la de aquellos profesores generacionalmente más próximos y con metodologías innovadoras.

\section{CONCLUSIONES}

1. En general, se observa una gran coincidencia de percepción por parte de los profesores a partir de los términos más frecuentes identificados en las tres Universidades. Mencionan en total 9 palabras clave en sus relatos, entre éstas, actividades, aprendizaje y formación/formar, las cuales sólo difieren en su frecuencia y en su relación con los principios orientadores actividad y participación, motivación y autoestima, aprendizajes significativos, globalización, personalización e interacción cuya profundidad y sentido depende del tiempo que las respectivas Universidades llevan implementando la enseñanza con enfoque en competencias.

2. Desde la perspectiva de la práctica docente, los profesores de la Universidad $A$, destacan en sus relatos las palabras actividades, aprendizaje, contenidos y experiencias. Los docentes de la universidad $\mathrm{B}$, refieren con mayor frecuencia los términos actividades, aprendizaje y formación/formar y, por último, los académicos de la universidad $\mathrm{C}$ se expresan por medio de las palabras trabajar, formación/formar, buscar/profesional y docencia. En consecuencia, parece claro que el fundamento del esfuerzo docente está en la didáctica, en los mecanismos de mejoramiento de la docencia y en la necesidad de habilitarse en este nuevo paradigma de enseñanza.

3. Desde la perspectiva del rol académico, los docentes de la Universidad A, declaran el propósito de guiar, educar, evaluar y estructurar. En la Universidad B, las palabras más frecuentes fueron guiar, enseñar y aprender y, los profesores de las Universidad C, se expresan con las palabras enseñar, estructurar, educar y aprender. Pudiéndose concluir que los profesores enfrentan una dicotomía, la de su labor docente que es principalmente la de enseñar y, la de su rol orientador que es esencialmente la de guiar a sus estudiantes para que ellos mismos logren el desarrollo de sus competencias.

4. Desde la perspectiva de los principios orientadores, en general se observa que los profesores de las Universidades A y C propician la globalización y, actividad y participación, mientras que en la Universidad B la atención se focaliza en los aprendizajes significativos. Categorías que representan sintéticamente cómo los profesores definen su función docente de pregrado.

5. Sintetizando las categorías menos mencionadas y en consecuencias, referidas como desafíos de la docencia, en la Universidad A, resultaron los términos enseñar, educar y evaluar, en la B, las palabras menos referidas fueron planificar, estructurar, capacitar y educar y, en la Universidad C, las palabras menos mencionadas resultaron ser aprender y capacitar. Las dos primeras (A y B) coincidiendo en su rol de educar y la segunda con la tercera (B y C) en la función de capacitar. Se puede inferir, en consecuencia, que los docentes internalizan en cada realidad el contexto situacional en el cual se encuentran, ellos mismos y sus estudiantes, pero sin embargo asumen que deben responder a la demanda institucional de poner en práctica un modelo de enseñanza más bien constructivista, el cual ha alcanzado en cada Universidad un estado de maduración diferente; la Universidad B, como la más longeva en el modelo seguido de la Universidad $\mathrm{A}$ y $\mathrm{C}$ respectivamente.

\section{REFERENCIAS}

Abela, D. A., Las Técnicas de Análisis de Contenido: Una Revisión Actualizada, Documentos de Trabajo, Fundación Centro de Estudios Andaluces, Sevilla, Serie Sociología (2002)

Alonso-Martin, P., La importancia y el nivel de desarrollo de las competencias en psicología, Psicología desde El Caribe, 25, 84-107 (2010) 
Ander-Egg, E., Métodos y Técnicas de Investigación Social. La Ciencia el Método y la Expresión del conocimiento científico, Editorial LUMUSA, Volumen II, Buenos Aires, Argentina (2004)

Ángel, D., Hermeneutics and research methods of Social Science, Estudios de Filosofía. (En línea: https://goo.gl/hToRiW). №44, 2011. Acceso: 10 de marzo de (2016)

Arango, A., Análisis de Datos Cualitativos, Medellín, Colombia (2011)

Baeza, M., De las metodologías cualitativas en investigación científico social. Diseño y uso de instrumentos en la producción de sentido, Editorial de la Universidad de Concepción, Concepción, Chile (2002)

Balcázar P., N. González-Arratia, G. Gurrola, A. Moysén, Investigación Cualitativa, México D.F., Universidad Autónoma del Estado de México (2013)

Barbier, J.M., Prácticas de Formación: Evaluación y Análisis. 1aㅡ Ed. Editorial Novedades Educativas, Buenos Aires, Argentina (1999)

Barragán, D., Hacia el Diálogo entre la Filosofía y Ciencias Sociales: dos posibilidades de Constitución de la Subjetividad Hermenéutica. En Fenomenología y Hermenéutica. Actas del I Congreso Internacional de Fenomenología y Hermenéutica. Editora Sylvia Izaguirre T., U. Andrés Bello, pp. 213- 223, Santiago de Chile (2008)

Bologna, The Bologna Declaration of June 1999. (En línea: https://goo.gl/155Abv). Acceso: 8 de junio de (2016)

Bunge, M., La investigación científica, Editorial Ariel, Barcelona, Hábitos de la Gente Altamente Efectiva, Editorial Piados, Buenos Aires, Argentina (1982)

Bustamante M., Método Formación - Acción en el desarrollo de Competencias, Cuadernos de Docencia Universitaria, 1(1), 1 - 74 (2008)

Bustamante, M., M. Grandón, M. Lapo, C. Oyarzún, Inteligibilidad de la docencia de pregrado: de la enseñanza por objetivos al desarrollo de competencias, Formación Universitaria, 9(5), 3-14 (2016)

Bustamante, M., C. Oyarzún, M. Grandón, y C. Abarza, Fundamentos de la Enseñanza por Competencias a Nivel de Postgrado en dos Universidades Públicas Chilenas, Formación Universitaria, 8(6), 23-30 (2015)

Camargo, I. y C. Pardo, Competencias docentes de profesores de pregrado: diseño y validación de un instrumento de evaluación, Universidad Católica de Colombia, Bogotá, Colombia, 441-455 (2008)

Cardoso, E., M. Cerecedo, y E. Vanegas, Las Competencias Docentes en los Programas de Posgrado en Administración: Un Estudio de Diagnóstico, doi: 10.4067/S0718-50062013000200006, Formación Universitaria, 6(2), 43-50 (2013)

Carrera, F., J. Cioduras, Identificación de las competencias digital del profesor universitario: un estudio exploratorio en el ámbito de las ciencias sociales, Revista de Docencia Universitaria, 10 (2), 273 - 298, Mayo - Agosto (2012)

Casado-Molina, A. y F. Cuadrado-Mendez, La reputación corporativa: Un nuevo enfoque de las competencias transversales en el EEES, Revista de Docencia Universitaria, 12, 353-371 (2014)

Castro A., Competencias administrativas y académicas en el profesorado de educación media superior, RMIE, 20(64), 263 - 294 (2015)

Checchia, B., Las Competencias del Docente Universitario (2009). (En línea: https://goo.gl/EqFkTe). Acceso: 10 de Marzo (2016)

Cisterna C., V., Soto y C. Rojas, Rediseño curricular en la Universidad de Concepción: La experiencia de las carreras de formación inicial docente, Calidad en la Educación, 44 (2016)

Clavijo D., El enfoque de competencias en la formación de abogados para el siglo XXI, Justicia, 27,1852012 (2015)

Coronado, E. y M., Estupiñán, Imaginarios sociales sobre el desempeño del docente universitario, ISSN: 1794-9998, Divers., Perspect. Psicol., 8(19), 73-84 (2012)

Crisol, E., Reseña de Cómo hicieron los sistemas educativos con mejor desempeño del mundo para alcanzar sus objetivos. Barber, Michael y Mourshed, Mona (2008), Revista de Currículum y Formación de Profesorado ISSN: 1138-414X. (En línea: https://goo.gl/CuxFgj). 14(2), 301-303 (2010), Acceso: 10 de Marzo (2016)

$\mathrm{CRUCH}$, Consejo de Rectores de las Universidades Chilenas. Manual para la implementación Sistema de créditos académicos trasferibles SCT-Chile, ISBN: 978-956-7581-05-4, Santiago de Chile, 1를. Ed., agosto (2013)

De Miguel, M. (Dir), I. Alfaro, P. Apodaca, J. Arias, E. García y A. Pérez, Adaptación de los planes de estudio al proceso de convergencia europea, Ediciones Universidad de Oviedo (2005) 
De Miguel, M. (Dir), I. Alfaro, P. Apodaca, J. Arias, E. García, C. Lobato y A. Pérez, Modalidades de enseñanza centradas en el desarrollo de competencias. Orientaciones para promover el cambio en el espacio europeo de educación superior, Ediciones Universidad de Oviedo (2005)

De Miguel, M. (Dir.), Metodologías de enseñanza y aprendizaje para el desarrollo de competencias. Orientaciones para el profesorado universitario ante el espacio europeo de educación superior, Revista Interuniversitaria de Formación del Profesorado, 20 (3), 316-319 (2006)

Denyer, M., J. Furnémont, R. Poulain, y G. Vanloubbeeck, Las competencias en la educación. Un balance, México D.F., Fondo de Cultura Económica (2007)

Díaz, C., P. Martínez, I. Roa y M. Sanhueza, Los docentes en la sociedad actual sus creencias y cogniciones pedagógicas respecto al proceso didáctico, Revista de la U. Bolivariana, 9(25) 421-436 (2010)

Diéguez, A., Tecnología y responsabilidad, Málaga, España, Revista de Filosofía, ISSN 0034-8244, 9, 189200 (1993)

Echeverría, R., Ontología del Lenguaje, Editorial Gránica (2007)

Eraut, M., Developing professional knowledge and competence, Londres, Falmer Press (1994)

Ettayebi, M., R. Opertti et Ph. Jonnaert (Dir.), Logique de competénces et developpment curriculaire. Débats, perspectives et alternative pour les systéms ëducatifs, Paris, L' Harmattan (2008)

Fernández, F., El análisis de contenido como ayuda metodológica para la investigación, Revista de la Universidad de Costa Rica, II (96), 35-54 (2002)

Fernandez-Elgueta, A. y C. Estrada-Goic, Percepción sobre las competencias especificas de formación profesional del cinesiólogo, Revista de la fundación educación médica, 19(3) (2016)

Gadamer, H., Verdad y método. Fundamentos de una hermenéutica filosófica, Editorial Sígueme, Salamanca, España (1998)

García, J., El enfoque socioformativo de formación en competencias: estructura y posibilidades para la gestión del currículum, Managua, UNI (2011)

García-Cabrero, B., J. Loredo, G. Carranza, Análisis de la práctica educativa de los docentes: pensamiento interacción y reflexión, doi https://doi.org/10.24320/redie.2017.19.2.1216, Revista Electrónica de Investigación Educativa, Especial, Vol. 10 (2008)

Germain, F., J. Vicente, C. Pérez-Rico y P. Villa, Formulación de preguntas de respuesta múltiples: un modelo de aprendizaje basado en competencias, Revista de la fundación educación médica, ISSN 20149840. (En línea: https://goo.gl/8MncDD). 19 (1), 27-38 (2016), Acceso: 10 de Marzo (2013)

Giménez, A., El papel de la gestión de centros educativos en un modelo de aprendizaje basado en competencias, Revista Páginas de Educación, 9(1) (2016)

Hernandez, R., C. Fernández, y L. Baptista, Metodología de la Investigación, México, Mac Graw Hill (2006)

Icarte, G. y H. Labate, Metodología para la revisión y actualización de un diseño curricular de una carrera universitaria incorporando conceptos de aprendizaje basado en competencias, For. Univ., 9(2) (2016)

Johnson-Laird, P., Thinking: Readings in Cognitive Science, Cambridge University Press (1977)

Johnson-Laird, P., The history of mental model (1983). (En línea: https://goo.gl/Bt4iQi). Acceso: 8 de junio de (2016)

Knust, R. y S. Gómez, La evaluación con enfoque por competencias: ¿se implementa realmente la evaluación por competencias? Experiencias en Holanda y diferentes países de América Latina, Revista Electrónica de Desarrollo de Competencias (REDEC), 1 (3), 104-125 (2009)

Lachiver, G., Abordando el cambio Curricular, La experiencia de la Universidad de Sherbrooke, Universidad de Santiago de Chile (2010)

Legault A., ¿Una enseñanza universitaria basada en competencias? ¿Por qué? ¿Cómo? Revista electrónica de desarrollo de competencias, REDEC, 5 (1), (2012)

López F., El análisis de contenido como método de investigación, Revista de Educación, Universidad de Huelva, 167-179 (2002)

Lopez, C., V. Benedito, M. Leon, El Enfoque de Competencias en la Formación Universitaria y su Impacto en la Evaluación: La Perspectiva de un Grupo de Profesionales Expertos en Pedagogía, Formación Universitaria, 9(4) 11-22 (2016) 
López, J. y E. Nelson, La deconstrucción curricular. Bogotá: Cooperativa Editorial Magisterio, ISBN: 9789582006013, p.176 (2003)

Mas-Torello, O. y P. Olmos-Rueda, El profesor Universitario en el espacio Europeo de Educación superior: La autopercepción de las competencias docentes actuales y orientaciones para su formación pedagógica, Revista Mexicana de Investigación Educativa, 21(69), (2016)

Morales, J. y M.Varela, El debate en torno al concepto de competencias, Investigación en Educación Medica, 4(13) (2015)

Muñoz, F., La Necesidad de Regular la Docencia Universitaria en Chile: una Propuesta de Lege Ferenda, doi: 10.4067/S0718-34372012000300019, Rev. Chil. Derecho, 39(3), 891-907 2012. (En línea: https://goo.gl/BsHxA9). Acceso: 18 de marzo de (2016)

Muñoz-Osuna, F., A. Medina-Revilla y M. Guillén-Lúgigo, Jerarquización de competencias genéricas basadas en las percepciones de docentes universitarios, Educación Química, 2(2) (2016)

Murcia, P., Los condicionantes: concertación e imposición en el desarrollo de la creatividad motriz. Apunts. №71. Barcelona, Generalitat de Catalunya, p. 30 (2003)

Ortiz O. y P. Lanceros, Claves de Hermenéutica para la filosofía, la cultura y sociedad. pp. 193-217, Universidad de Deusto, Bilbao, España (2005)

Patiño-Garzón, L. y M. Rojas-Betancur, Subjetividad y subjetivación de las prácticas pedagógicas en la Universidad Scielo, 93-105 (2009)

Pavié, A., Formación docente: hacia una definición del concepto de competencia profesional docente, REIFOR, 14 (1), 67-80 (2011)

Pedraja, L., Desafíos para el Profesorado en la Sociedad del Conocimiento, Ingeniare. Rev. Chil. Ing., 20(1), 136-144 (2012)

Perrenoud, P., La Formación de los Docentes en el Siglo XXI, Revista de Tecnología Educativa, Santiago de Chile, XIV (3) 503-523 (2001)

Pey, R. y S. Chauriye, Innovación Curricular en las Universidades del Consejo de Rectores 2000-2010, Santiago de Chile (2011)

Popper, K., Conjeturas y refutaciones: el desarrollo del conocimiento científico, Paidós lbérica Ediciones, S.A. (1994)

Popper, K., Conocimiento objetivo: un enfoque evolucionista. Editorial Tecnos (2005)

Puig, J. y X. García, Competencia en autonomía e iniciativa personal, Madrid, Alianza (2007)

Robles-Haros, I. y E. Esteves-Nenninger, Enfoque por competencias: Problemática didácticas que enfrentan el profesorado, Revista Electronica Educare, 20(1) (2016)

Ruay, R., El rol del docente en el contexto actual, Revista Electrónica de Desarrollo de Competencias (REDEC), 2 (6), (2010)

Santander, P., Por qué y cómo hacer análisis de discurso, Cinta moebio, 41, 207-224 (2011)

Sutcliffe, N., S. Chan y M. Nakayama, Competency Based MSIS Curriculum, Journal of Information System Education 16(3), 301 (2005)

Turcio-Ortega, D. y J. Palacios-Alquisira, Experiencias en la enseñanza experimental basada en competencias, Educación Química 26(1), (2015)

Vattimo G., Ética de la interpretación, Barcelona, Paidós, 59-62, (1991)

Viquez-Moreno, D., J. Valenzuela-González y M. Compeán-Aguilar, Identificación de competencias transversales para reformas curriculares: El caso de la multiculturalidad, Rev. Electronica Educare 19(2), (2015)

Zabalza, M., Articulación y rediseño curricular: el eterno desafío institucional, Revista de Docencia Universitaria 10 (3), octubre-diciembre, ISSN: 1887-4592, 17 - 48 (2012) 
